\title{
Synthesis and Properties of Charged Copolypeptides Membranes as Biodegradable Materials
}

\author{
Toshio Hayashi, Yasuko Irzuka, ${ }^{*}$ Masanao Oya, \\ and Makoto IWATSUKI** \\ Research Center for Biomedical Engineering, Kyoto University, \\ Sakyo-ku, Kyoto 606, Japan \\ * Department of Biological Science, Faculty of Engineering, \\ Gunma University, Kiryu 376, Japan \\ ** Ajinomoto Co., Inc., Chuo-ku, Tokyo 104, Japan
}

(Received September 7, 1992)

\begin{abstract}
Three-component random copolypeptides consisting of $N$-hydroxyalkyl Lglutamine, L-aspartic acid, and L-lysine, related two component random copolymers and homopolymers, were prepared by aminolysis with 2-amino-1-ethanol [AE] or 5-amino-1-pentanol [APe], followed by crosslinking reaction with 1,8-octamethylenediamine [OMDA] on starting polymer membranes consisting of $\gamma$-methyl L-glutamate [E], L-aspartic acid [D], and L-lysine [K]. All water soluble samples prepared without OMDA were degraded by random chain fracture with papain. The effective crosslink density was proportional to the content of the crosslinker [OMDA] in the reaction mixture. Tensile properties of these hydrophilic membranes were highly dependent on the swelling ratio in a pseudo-extracellular fluid [PECF], hydrophobicity of side chains. The effective charge density of membranes, and their behavior were typical of elastomers. A higher rate of water permeability was obtained with charged membranes than non-charged and/or compensated charged membranes with the same order of swelling ratio in PECF. Biodegradation of the samples in vitro by papain indicated that degradation could be regarded as a bulk rather than surface phenomenon. The rate of degradation was also dependent on the swelling ratio of membranes, as well as on the hydrophobicity and effective charge density of side chains of the samples.
\end{abstract}

KEY WORDS Three Component Random Copolypeptide / Charged Membrane / Enzymatic Degradation / Papain / Swelling Ratio / Tensile Property /

Many proteins contain both anionic and cationic groups in their molecules, and thus it is interesting to investigate conformations as well as membrane properties of copolypeptides carrying both negative and positive charges in the side chains of the same molecules from basic points of view for biomedical materials. ${ }^{1}$

In this study, we prepared three component random copolypeptides [DKE(A)] consisting of L-aspartic acid [D], L-lysine [K], and $N$-hydroxyalkyl L-glutamine $[\mathrm{E}(\mathrm{A})]$ as well as the corresponding two-component random copolypeptides, copoly(L-aspartic acid $/ N$ hydroxyalkyl L-glutamine) $[\mathrm{DE}(\mathrm{A})]$ and copoly(L-lysine/ $N$-hydroxyalkyl L-glutamine) [KE(A)], and poly( $N$-hydroxyalkyl L-glutamine) [PHAG] and investigated the relation between bulk structure and membrane properties, such as the swelling ratio in a pseudoextracellular fluid $[\mathrm{PECF}],^{2}$ tensile properties, water vapor permeability, and enzymatic degradation behavior in vitro of these membranes in PECF. 


\section{EXPERIMENTAL}

\section{Materials}

Synthesis of Copolypeptides. Three-component random copolypeptide, $\operatorname{copoly}(\beta$-benzyl L-aspartate $/ \varepsilon-N$-benzyloxycarbonyl L-lysine $/ \gamma$ methyl L-glutamate) [DKE], two-component random copolymers, $\operatorname{copoly}(\beta$-benzyl L-aspartate $/ \gamma$-methyl L-glutamate) [DE], and copoly( $\varepsilon$ - $N$-benzyloxycarbonyl L-lysine $/ \gamma$-methyl Lglutamate) [KE], and the corresponding homopolymer, poly $(\gamma$-methyl L-glutamate) [PMLG] with various molecular weights, were synthesized by the $\mathrm{N}$-carboxyanhydrides [NCA] method. NCA of L-amino acids were prepared by the phosgenation of the corresponding L-amino acids in tetrahydrofuran [THF] and purified by multiple recrystallization. $^{3}$

Solutions $(0.1 \mathrm{M}, c a .2 \mathrm{wt} \%)$ of a mixture of $\beta$-benzyl L-aspartate $N$-carboxyanhydride [D-NCA], $\varepsilon-N$-benzyloxycarbonyl L-lysine $N$ carboxyanhydride [K-NCA], and $\gamma$-methyl L-glutamate $N$-carboxyanhydride [E-NCA] in a $1: 1(\mathrm{v} / \mathrm{v})$ mixture of dioxane and methylene dichloride were prepared and polymerization was initiated with triethylamine [TEA], in which the NCA monomers to TEA molar ratio of 25. All polymer samples were precipitated by adding about 4 times the amount of methanol in volume including $5 \mathrm{vol} \%$ of $0.1 \mathrm{~N}$ $\mathrm{HCl}$ to the polymer solution at $4^{\circ} \mathrm{C}$. The precipitated products were washed in methanol and dried under reduced pressure at $50^{\circ} \mathrm{C}$. The preparative data of polymer samples are listed in Table I.

Papain (3.5m Anson $\mu \mathrm{g} \mathrm{mg}^{-1}$, No. 7144) was purchased from Nacalai Tesque Co. (Kyoto, Japan), and used without further recrystallization. Papain was activated in the PECF solution as proposed by Homsey ${ }^{2}$ (see Table II) at $\mathrm{pH} 7.4$ with $0.01 \mathrm{M}$ cystein and $0.01 \mathrm{M}$ EDTA. ${ }^{4}$ The other chemicals were of analytical grade.

Preparation of Hydrophilic Polymer Membranes. The debenzylation of $\beta$-benzyl L-
Table I. Copolymerization of random copolypeptides

\begin{tabular}{|c|c|c|c|c|}
\hline \multirow{2}{*}{ Sample code } & \multicolumn{3}{|c|}{ Composition/mol\% } & \multirow{2}{*}{$\frac{[\eta] / \mathrm{dl} \mathrm{g}^{-1}}{\left(\mathrm{DCA}, 25^{\circ} \mathrm{C}\right)}$} \\
\hline & $\mathrm{D}$ & K & $\mathrm{E}$ & \\
\hline DKE-1 & 9.8 & 9.6 & 80.6 & 1.45 \\
\hline DE-1 & 9.5 & 0.0 & 90.5 & 1.66 \\
\hline KE-1 & 0.0 & 10.2 & 89.8 & 0.98 \\
\hline PMLG-1 & 0.0 & 0.0 & 100.0 & 2.33 \\
\hline
\end{tabular}

Table II. Pseudo-extracellular fluid (PECF) $\left(\mathrm{NaHCO}_{3}, \mathrm{~K}_{2} \mathrm{HPO}_{4}, \mathrm{NaCl}, \mathrm{KCl}\right)$

\begin{tabular}{lcr}
\hline & Physiological & PECF \\
\cline { 2 - 3 } Ion & \multicolumn{2}{c}{ Concentration/meq l $^{-1}$} \\
\hline $\mathrm{Na}$ & 145 & 145 \\
$\mathrm{~K}$ & 5 & 5 \\
$\mathrm{Cl}$ & 113 & 118 \\
$\mathrm{HCO}_{3}$ & 30 & 30 \\
$\mathrm{HPO}_{4}$ & 2 & 2 \\
\hline
\end{tabular}

aspartate [D] as well as decarbobenzylation of $\varepsilon$ - $N$-benzyloxycarbonyl L-lysine [K] residues in copolymers were performed by anhydrous $\mathrm{HBr}$ treatment according to the method of Idelson and Blout. ${ }^{5}$ After a membrane of $c a$. $100 \mu \mathrm{m}$ in thickness cast from an appropriate solvent was immersed in a mixture of 2amino-1-ethanol [AE] and the crosslinker, 1,8-octamethylenediamine [OMDA], as well as a mixture of 5-amino-1-pentanol [APe], 1pentanol, and OMDA, at 58 to $62^{\circ} \mathrm{C}$ for $48 \mathrm{~h}$, the membrane was washed with pure water, ethyl ether, and stored in ethanol. Corresponding water soluble samples were prepared in the same way but without the crosslinker OMDA.

\section{Measurements}

Molecular Characterizations. The intrinsic viscosity $[\eta]$ of starting polymers was determined in dichloroacetic acid [DCA] at $25.0^{\circ} \mathrm{C}$ using an Ubbelohde-type viscometer and is listed in Table I. Molecular weights of the water soluble samples after deblocking reac- 
Table III. Preparative data on water soluble copolypeptides

\begin{tabular}{lcccc}
\hline & & {$[\eta] / \mathrm{dl} \mathrm{g}^{-1}$} & & \multirow{2}{*}{$\boldsymbol{M}_{\boldsymbol{w}}$} \\
\cline { 3 - 3 } Sample code & Starting polymer & PECF, 37 $7^{\circ} \mathrm{C}$ & & \\
\hline DKE(E)-10 & DKE-1 & 0.215 & 26,200 & -60 \\
DKE(Pe)-10 & DKE-1 & & & -530 \\
DE(E)-10 & DE-1 & 0.228 & 28,800 & -20 \\
DE(Pe)-10 & DE-1 & & & -510 \\
KE(E)-10 & KE-1 & 0.140 & 13,400 & -30 \\
KE(Pe)-10 & KE-1 & & & -520 \\
PHEG-10 & PMLG-1 & 0.320 & 69,400 & 0 \\
PHPeG-10 & PMLG-1 & & & -510 \\
\hline
\end{tabular}

tion were estimated at $\mathrm{pH} 7.4$ and $37.0{ }^{\circ} \mathrm{C}$ in PECF by sedimentation equilibrium using a MOM type 3170-b ultracentrifuge equipped with a Reyleigh interference optical system and a $12 \mathrm{~mm}$ double sector cell. ${ }^{6}$ Intrinsic viscosities were measured with an Ubbelohdetype viscometer on the same buffer at $\mathrm{pH} 7.4$ and $37.0^{\circ} \mathrm{C}$. The preparative data of the water soluble samples are summarized in Table III.

The chain conformations of these copolymers in PECF were examined by optical rotatory dispersion [ORD] measurements. The Moffitt-Yang parameter bo was evaluated from ORD data obtained with Yanagimoto OR-100 Type spectropolarimeter, using a tungsten lamp at $37.0^{\circ} \mathrm{C}$. Table III also includes experimental data of bo for these copolymers at $\mathrm{pH} 7.4$ in PECF.

The molecular weight distribution curves of these samples were investigated by gel permeation chromatography [GPC] on a Toyo-Soda High-Speed Liquid Chromatograph HLC-803D equipped with TSK-Gel Type G-4000SW, C-No. SW46A0015 in PECF at $25^{\circ} \mathrm{C}$.

Physical Properties of Hydrophilic Membranes. The swelling ratio $q$ in PECF was determined by equilibrating the membrane in PECF solution at $37.0^{\circ} \mathrm{C}$. The membrane was removed, blotted to remove surface PECF and weighed until constant weight was noted. The membrane was dried in a vacuum oven. $q$ was defined as the ratio of the weight of the PECF swelling hydrophilic membrane to that of the dried one.

Tensile properties of hydrophilic membranes were measured by a Tensilon UTM-II20 (Toyo-Boldwin Co., Tokyo, Japan) using standard techniques in PECF at $25.0^{\circ} \mathrm{C}$. All samples were tested at an elongation rate of $40 \%$ per minute.

Water vapor permeation through the membranes was measured with a cylindrical glass cell $^{7}$ at $37.0^{\circ} \mathrm{C}$. The exposed membrane area was $12.57 \mathrm{~cm}^{2}$.

Biodegradation of Hydrophilic Membranes in Vitro. Enzymatic degradation studies in vitro were carried out using papain. Although papain is a general plant thiol endopeptidase, it has preference for peptide bonds where the amino acid residue of the carbonyl group is lysine, glutamine, or arginine and where the amino acid is joined on either side by amino acids with hydrophobic side chains. ${ }^{8}$ A series of the crosslinked hydrophilic membranes was exposed to PECF solution at $\mathrm{pH} 7.4$ and $37^{\circ} \mathrm{C}$. These membranes were removed from the enzyme solution at appropriate time intervals, weighed, and then vacuum dried at $60^{\circ} \mathrm{C}$ to constant weights. 


\section{RESULTS AND DISCUSSION}

\section{Type of Degradation}

To determine whether random degradation of the main-chain of a polypeptide is dominant in the reaction with papain, GPC analyses of partially degraded polypeptides were carried out. Figure 1 illustrates examples of GPC curves for water soluble polypeptide samples; copoly(L-aspartic acid/L-lysine $/ N$ hydroxyethyl L-glutamine) [DKE(E)-10] and $\operatorname{poly}(N$-hydroxyethyl L-glutamine) [PHEG10]. From Figure 1, it may concluded that each sample is dominantly degraded by a random main-chain cleavage as in the case of the degradation of PHEG by endopeptidases such as bromelain, chymotrypsin, ficin or pepsin. ${ }^{9,10}$

\section{Swelling Ratio of Hydrophilic Membranes in $P E C F$}

The swelling ratio $q$ of hydrophilic polymers in a solvent is determined by interaction energy between the solvent molecules and polymer segments as well as the elastic energy (crosslink density) for a solvent-swollen poly-

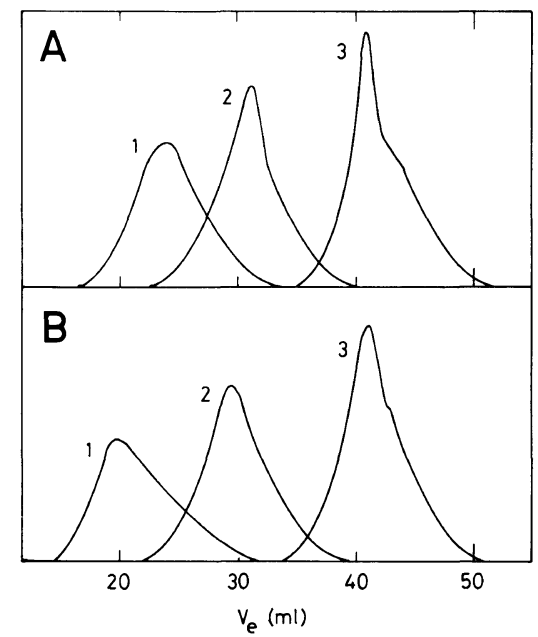

Figure 1. GPC elution curves for reaction products of polypeptides at $\mathrm{pH} 7.4$ and $25^{\circ} \mathrm{C}$ in $\mathrm{PECF}$ by papain. $[E]=5.1 \times 10^{-6} \mathrm{M}$. (A) DKE(E)-10 for; (1) original, (2) $30 \mathrm{~min}$, and (3) $300 \mathrm{~min}$ of digestion, and (B) PHEG-10 for; (1) original, (2) $45 \mathrm{~min}$, and (3) $450 \mathrm{~min}$ of digestion. mer.

Crosslink density has not been precisely determined because of the uncertainty in the relative reactivities of $\mathrm{AE}$ or $\mathrm{APe}$ and OMDA, and also because estimation of the fraction of the reacted diamine molecules which form effective crosslinks is difficult. The effect of crosslinker OMDA molar percent $(\mathrm{mol} \%)$ in the reaction on the swelling ratio $q$ of the crosslinked membranes in PECF is shown in Figure 2. The $q$ value in PECF decreases with increasing OMDA mol\% in the reaction mixture.

When the swelling ratio $q$ is quite large, it is given by the following equation according to the rubber elasticity theory. ${ }^{11}$

$$
q^{5 / 3}=\left(v M_{\mathrm{c}}\right)\left(1-2 M_{\mathrm{c}} / M\right)^{-1}\left(1 / 2-\chi_{1}\right) / V_{1}
$$

where $M_{\mathrm{c}}$ is the molecular weight per crosslinked unit, $M$ the primary molecular weight, $v$ the specific volume of polymer, $V_{1}$ the molar volume of solvent and $\chi_{1}$ the interaction parameter. The factor $\left(1-2 M_{\mathrm{c}} / M\right)$ expresses the correction for network inperfections resulting from chain ends. For a quite high molecular weight polymer chain, it reduces to unity.

As the effective crosslink density, $f_{\mathrm{c}}$ is

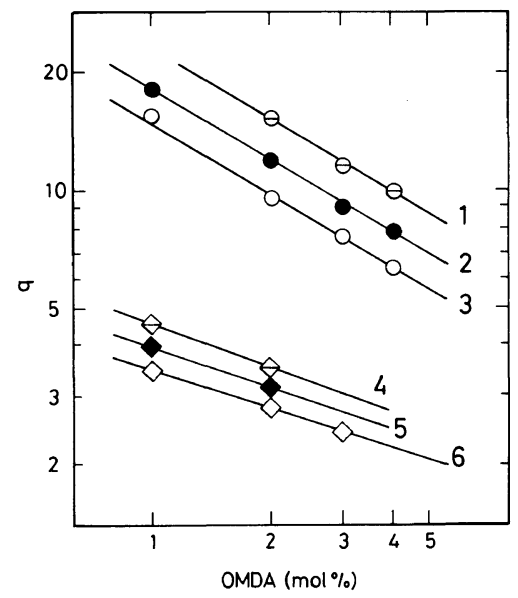

Figure 2. Swelling ratio $(q)$ of hydrophilic membranes at $25^{\circ} \mathrm{C}$ as a function of molar percent of OMDA. (1) $\mathrm{DE}(\mathrm{E})-1 n$; (2) DKE(E)-1 $n$; (3) PHEG-1 $n$; (4) DE(Pe)-1n; (5) DKE(Pe)-l $n$; (6) PHPeG-1n. 
proportional to the value of $M_{\mathrm{o}} / M_{\mathrm{c}}$ where $M_{\mathrm{o}}$ is the molecular weight of the repeat unit (monomeric unit), eq 1 may be simplified as

$$
q^{5 / 3}=\left(v M_{\mathrm{o}}\right)\left(1 / 2-\chi_{1}\right) / V_{1} f_{\mathrm{c}}
$$

Thus, $\log q$ is in linear relation with $\log f_{\mathrm{c}}$ with the slope as $-3 / 5$.

Figure 2 illustrates the effect of OMDA $\mathrm{mol} \%$ in the reaction mixture on the $q$ value of the crosslinked hydrophilic membranes. The $q$ value decreases with increasing OMDA $\mathrm{mol} \%$ in the reaction mixture. The slope of the $\log -\log$ plots in a series of hydrophilic membranes of poly $(N$-hydroxyethyl L-glutamine) [PHEG-1 $n ; n=1,2,3$, and 4 which denotes OMDA mol\% in the reaction mixture], copoly(L-aspartic acid/ $N$-hydroxyethyl L-glutamine) $[\mathrm{DE}(\mathrm{E})-1 n ; n=2,3$, and 4$]$, and copoly(L-aspartic acid/L-lysine/ $N$-hydroxyethyl L-glutamine) [DKE(E)-1 $n ; n=1,2,3$, and 4], respectively, which exist mainly in random coil conformation with the corresponding water soluble samples (see Table III), has the value of $-3 / 5$ as predicted in eq 2 . The effective crosslink density $f_{\mathrm{c}}$ is proportional to the crosslinker OMDA mol\% in the reaction mixture. $q$ obtained with $\mathrm{DE}(\mathrm{E})-1 n$, negatively charged membranes, were higher than those obtained with the compensated-charged membranes, $\operatorname{DKE}(\mathrm{E})-1 n$, as well as non-charged membranes, PHEG- $1 n$ of the same order of OMDA mol\%, indicating that $q$ depends on the net charge in membranes. On the other hand, $\operatorname{poly}(N$-hydroxypentyl L-glutamine) [PHPeG-1 $n: n=1,2$, and 3], copoly(L-aspartic acid/ $N$-hydroxypentyl L-glutamine) $[\mathrm{DE}(\mathrm{Pe})$ $1 n ; n=1$ and 2], and copoly(L-aspartic acid/ L-lysine/ $N$-hydroxypentyl L-glutamine) [DKE(Pe)-1n: $n=1$ and 2], which exist in $\alpha$-helix conformation with the corresponding water soluble samples (see Table III), cannot be dealt with in terms of the rubber elasticity theory in which the slope is less than $-3 / 5$ with lower $q$ values. This suggests that the theory given in eq 1 can only be applied to hydrophilic membranes which contain ran- dom coil region having enough segmental mobility. ${ }^{12}$

\section{Tensile Properties of Hydrophilic Membranes} in PECF

The tensile properties of hydrophilic membranes are highly dependent on the $q$ values in PECF. Further, elastomeric membranes are highly suited to biomedical applications, such as membranes for artificial organs, reconstructive prosthesis, and cosmesis.

Figure 3 illustrates the stress-strain curves of some examples of hydrophilic membranes in PECF at $25^{\circ} \mathrm{C}$. In Figure 3, while $\mathrm{DE}(\mathrm{E})$ 12 and PHEG-12 membranes gave lower strength, DKE(E)-12 and DKE(E)-13 attained higher strength with a moderate modulus. Table IV lists the experimental findings of Young's modulus $E$ at an elongation of $1 \%$, the tensile strength $\sigma_{\mathrm{B}}$ and elongation $\varepsilon_{\mathrm{B}}$ at the breakage point with the $q$ values for hydrophilic membranes with various OMDA $\mathrm{mol} \%$ in PECF.

Tensile properties of DKE(E)-12 and DKE(Pe)-11 membranes were more than PHEG-12 and PHPeG-11, respectively, even when $q$

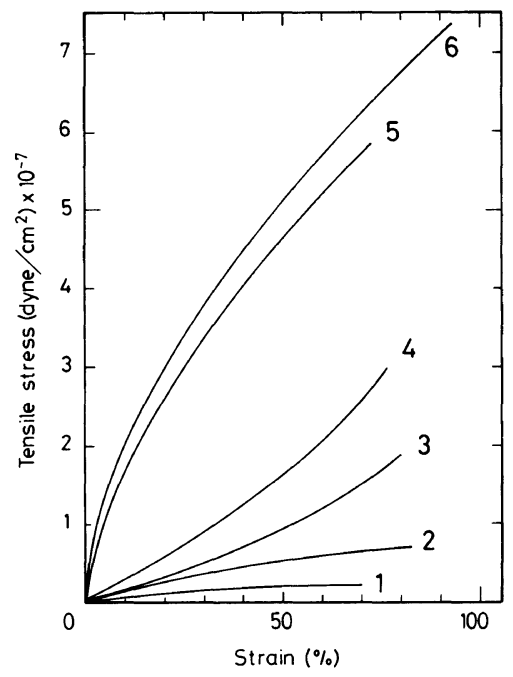

Figure 3. Stress-strain curves of hydrophilic membranes at $25^{\circ} \mathrm{C}$ in PECF. (1) DE(E)-12; (2) PHEG-12; (3) DKE(E)-12; (4) DKE(E)-13; (5) PHPeG-11; (6) DKE(Pe)11 . 
Table IV. Mechanical properties of hydrogels at $25.0^{\circ} \mathrm{C}$ in PECF

\begin{tabular}{|c|c|c|c|c|c|}
\hline \multirow{2}{*}{ Sample code } & OMDA & $q$ & $E \times 10^{7}$ & $\sigma_{\mathrm{B}} \times 10^{7}$ & $\varepsilon_{\mathbf{B}}$ \\
\hline & $\mathrm{mol} \%$ & $W_{\mathbf{w}} / W_{\mathrm{D}}$ & $\mathrm{dyn} / \mathrm{cm}^{-2}$ & $\mathrm{dyn} / \mathrm{cm}^{-2}$ & $\%$ \\
\hline DKE(E)-12 & 2 & 11.8 & 1.50 & 1.85 & 80 \\
\hline DKE(E)-13 & 3 & 8.9 & 2.70 & 3.00 & 77 \\
\hline $\mathrm{DE}(\mathrm{E})-12$ & 2 & 14.5 & 0.50 & 0.25 & 70 \\
\hline PHEG-12 & 2 & 9.5 & 1.30 & 0.65 & 83 \\
\hline PHEG-13 & 3 & 7.6 & 1.85 & 1.60 & 90 \\
\hline $\mathrm{DKE}(\mathrm{Pe})-11$ & 1 & 3.9 & 38.0 & 7.35 & 93 \\
\hline $\mathrm{DE}(\mathrm{Pe})-12$ & 2 & 3.5 & 18.5 & 5.05 & 75 \\
\hline PHPeG-11 & 1 & 3.3 & 21.0 & 5.80 & 73 \\
\hline
\end{tabular}

of $\operatorname{DKE}(\mathrm{E})-12$ and $\operatorname{DKE}(\mathrm{Pe})-11$, the compensated-charged membranes, were more than the corresponding non-charged membranes, PHEG-12 and PHPeG-11, showing charge interactions between cation and anion in the molecular chains to affect the tensile strength of the hydrophilic membranes. Further, it is again pointed out that $q$ values for membranes containing $N$-hydroxypentyl L-glutamine $[\mathrm{E}(\mathrm{Pe})]$ component become much lower than those for membranes containing $N$-hydroxyethyl L-glutamine $[\mathrm{E}(\mathrm{E})]$ component. The hydrophobic nature of glutamine side chain increases from $\mathrm{E}(\mathrm{E})$ to $\mathrm{E}(\mathrm{Pe})$. Lotan et al. have shown that helical content increases with increasing length of hydrocarbon of side chain in aqueous solution, ${ }^{13}$ and that PHPeG exists almost completely in $\alpha$-helix conformation, while PHEG exists in random coil conformation. Thus, $q$ may be affected by molecular conformation. Furthermore, it is pointed out from Figure 3 and Table IV that the hydrophobic nature of the glutamine side chains affects the mechanical properties of membranes.

\section{Water Vapor Permeability of Membranes}

Figure 4 illustrates the relation between the rate of water vapor permeability $V_{\mathrm{f}}\left(\mathrm{g} \mathrm{m}^{-2}\right.$. day) of PECF and the $q$ value for membranes at $37.0^{\circ} \mathrm{C}$. It is also shown that the $V_{\mathrm{f}}$ value is highly dependent on the $q$ value in PECF. Further, it may be judged in Figure 4 that the

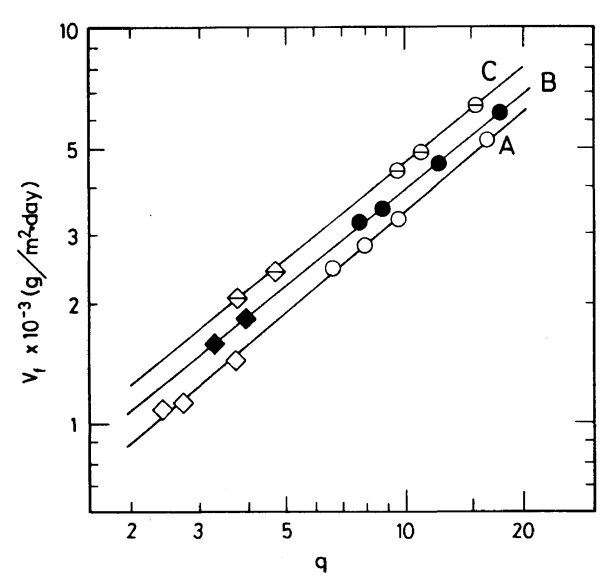

Figure 4. Rates of water vapor permeability $\left(V_{\mathrm{f}}\right)$ of hydrophilic membranes as a function of the swelling ratio (q) at $\mathrm{pH} 7.4$ and $37.0^{\circ} \mathrm{C}$ in PECF. (A) (O) PHEG- $1 n$ and $(\diamond)$ PHPeG- $1 n$; (B) (O) DKE(E)-1n and $(\diamond)$ PHPeG-1; (C) (O) DE(E)-1n and $(\diamond) \mathrm{DE}(\mathrm{Pe})-1 n$.

$V_{\mathrm{f}}$ value of charged membranes, $\mathrm{DE}(\mathrm{E})-1 n$ and $\mathrm{DE}(\mathrm{Pe})-1 n$, is more than those of the compensated-charged or non-charged membranes, $\mathrm{DKE}(\mathrm{E})-1 n$ and $\mathrm{DKE}(\mathrm{Pe})-1 n$ as well as PHEG- $1 n$ and PHPeG- $1 n$, respectively, at the same $q$ values, indicating that the net charge in membranes affects the state of water in membranes.

\section{Biodegradation of Membranes in Vitro}

Pre-weighed crosslinked sample membranes were exposed to $0.1 \mathrm{mg}$ of papain in $1 \mathrm{ml}$ of the appropriate activator solution at $37.0^{\circ} \mathrm{C}$, and experimental results obtained with DKE- 


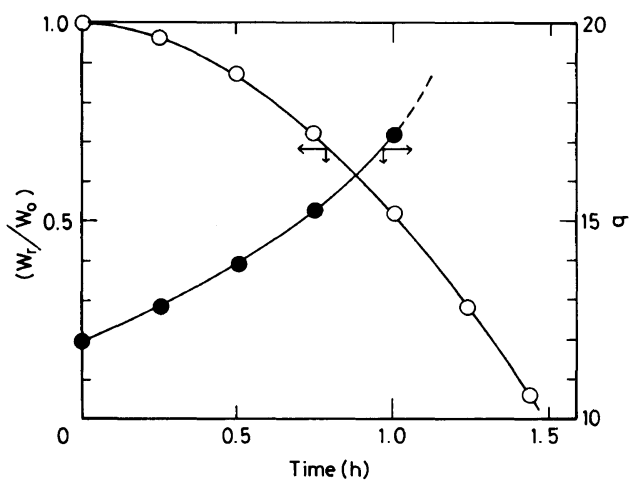

Figure 5. Dry weight ratio $\left(W_{\mathrm{r}} / W_{\mathrm{o}}\right)$ and swelling ratio (q) of PECF for DKE(E)-12 membrane as a function of papain digestion time (h) at $\mathrm{pH} 7.4$ and $37.0^{\circ} \mathrm{C}$.

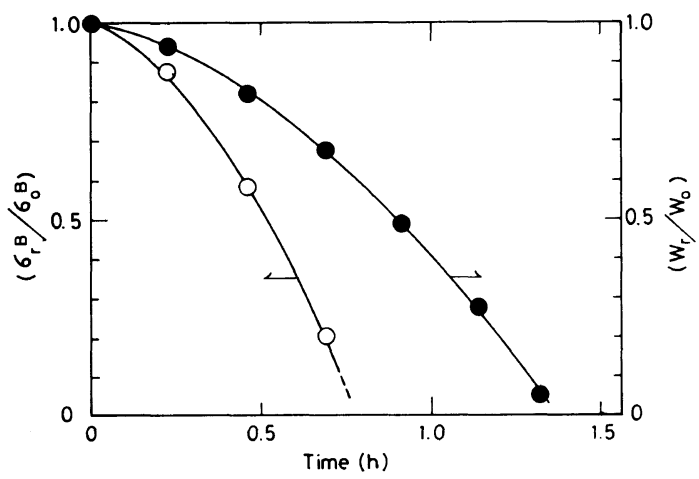

Figure 6. Dry weight ratio $\left(W_{\mathrm{r}} / W_{\mathrm{o}}\right)$ and relative tensile strength at break $\left(\sigma_{\mathrm{B}, \mathrm{r}} / \sigma_{\mathrm{B}, \mathrm{o}}\right)$ of $\mathrm{DKE}(\mathrm{E})-12$ membranes as a function of papain digestion time (h) at $\mathrm{pH} 7.4$ and $37.0^{\circ} \mathrm{C}$.

(E)-12 membrane are illustrated in Figure 5. As shown in Figure 5, with papain digestion an immediate increase in the swelling ratio $q$ of the $\mathrm{DKE}(\mathrm{E})-12$ membrane was observed, while weight loss occurred slightly more slowly. An endo-peptidase must make two incisions in a chain segment to produce a soluble fragment, but a single cleavage will decrease the effective crosslink density.

It is also important to know exactly the change of the remaining tensile strength of membranes under enzymatic digestion from the applicational point of view. Figure 6 illustrates the relative tensile strength $\left(\sigma_{\mathrm{B}, \mathrm{r}} /\right.$ $\left.\sigma_{\mathrm{B}, \mathrm{o}}\right)$ and dry weight ratio $\left(W_{\mathrm{r}} / W_{\mathrm{o}}\right)$ for DKE-

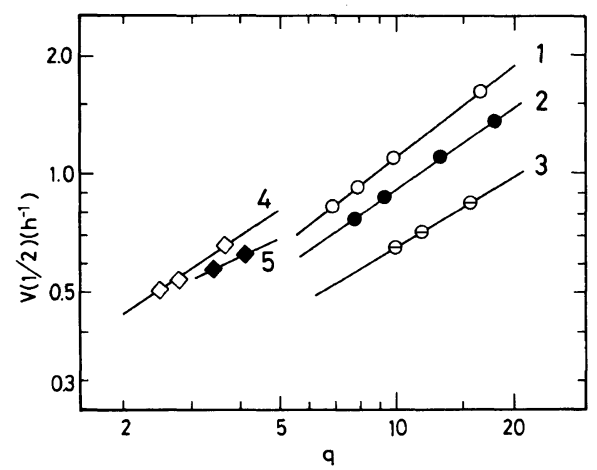

Figure 7. Rate of papain digestion $V(1 / 2)$ of hydrogels at $\mathrm{pH} 7.4$ and $37.0^{\circ} \mathrm{C}$ in PECF as a function of the swelling ratio (q): (1) PHEG- $1 n$; (2) DKE(E)- $1 n$; (3) $\mathrm{DE}(\mathrm{E})-1 n$; (4) PHPeG-1n; (5) DKE(Pe)-1n.

(E)-12 membrane as a function of papain digestion time at $37.0^{\circ} \mathrm{C}$ and $\mathrm{pH} 7.4$ in PECF. It is clearly shown that the order of decrease in tensile strength compared to that of mass for papain digestion is rather high, suggesting that the cleavage of crosslink occurs practically under enzymatic digestion.

Figure 7 summarizes the rate of papain digestion $V(1 / 2)(\mathrm{h})$ as a function of the $q$ value of PECF for these membranes. $V(1 / 2)$ is defined as the reciprocal of the time required for the sample weight to be reduced to onehalf its initial value. It is also clearly shown that the order of the degradation rate among membranes is as follows: $\mathrm{PHPeG}>\mathrm{DKE}(\mathrm{Pe})$ and $\mathrm{PHEG}>\mathrm{DKE}(\mathrm{E})>\mathrm{DE}(\mathrm{E})$ at the same $q$ value for each membrane, respectively.

In conclusion, the effective crosslink density is proportional to the mol\% of the crosslinker (OMDA) in the reaction mixture. $q$ values were highly dependent on the net charge in membranes. Tensile properties of these hydrophilic membranes depended on the $q$ value in PECF, charged interactions between cation and anion in the molecular chain, and the molecular conformation of chains in membranes, and their behavior was typical of an elastomer.

The biodegradation of these hydrophilic membranes by papain in vitro indicated that 
the degradation could be regarded as a bulk rather than a surface phenomenon. The rate of degradation of sample was also highly dependent on the $q$ value of membranes, as well as on net charge of the side chains of samples.

\section{REFERENCES}

1. J. M. Anderson, K. L. Spilizewski, and A. Hiltner, in "Biocompatibility of Tissue Analogs," D. F. Williams, Ed., CRC Press, N. W. Boca Raton, Florida, 1985, p 68.

2. C. A. Homsey, J. Biomed. Mater. Res., 4, 341 (1971).

3. T. Hayashi, E. Nakanishi, and A. Nakajima, Kobunshi Ronbunshu, 43, 633 (1986).

4. J. Lowbridge and J. S. Fruton, J. Biol. Chem., 249, 6754 (1974).
5. M. Idelson and E. R. Blout, J. Am. Chem. Soc., 80, 4631 (1958).

6. T. Hayashi, G. W. Chen, and A. Nakajima, Polym. J., 16, 739 (1984).

7. T. Hayashi, K. Takeshima, E. Kobatake, and A. Nakajima, Kobunshi Ronbunshu, 42, 777 (1985).

8. H. R. Dickinson and A. Hiltner, J. Biomed. Mater. Res., 15, 591 (1981).

9. T. Hayashi, K. Itoh, and A. Nakajima, Polym. J., 1, 524 (1970).

10. T. Hayashi, M. Iwatsuki, and M. Oya, J. Appl. Polym. Sci., 39, 1803 (1990).

11. P. J. Flory, in "Principle of Polymer Chemistry," Cornell Univ. Press, Ithaca, N.Y. and London, 1953, p 576.

12. T. Hayashi, E. Nakanishi, and A. Nakajima, Polym. J., 19, 1025 (1987).

13. N. L. Lotan, A. Yaron, A. Berger, and M. Sela, Biopolymers, 3, 625 (1965).

14. J. Hubacek, K. Kliment, J. Dusek, and J. Hubacek, J. Biomed. Mater. Res., 1, 387 (1967). 